\title{
Use of Orthogonal Array to Study the Effect of Various Parameters on Liquid Metal based Microchannel Cooling
}

\author{
Deewakar Sharma \\ CSIR-Central Scientific \\ Instruments Organisation \\ Chandigarh India, 160030
}

\author{
Harry Garg \\ CSIR-Central Scientific \\ Instruments Organisation \\ Chandigarh, India, 160030
}

\author{
P. P. Bajpai \\ CSIR-Central Scientific \\ Instruments Organisation \\ Chandigarh, India, 160030
}

\begin{abstract}
With increase in demand for efficient cooling technologies in electronic systems, use of microchannels with liquid metals as cooling medium has gained significant attention. To analyze the effect of various geometrical parameters on microchannel performance with such coolant, Taguchi Orthogonal Arrays are used in this study. To replicate the results for simulation, external noise is introduced in two parameters, channel width and depth. In addition, channel wall and base thickness along with type of material affecting the performance is also analyzed. Results show that channel width, height and substrate thickness at the base are the prime factors of concern. In addition, interactive effect of wall and base thickness is also found to affect the performance significantly and their low values for optimum performance is recommended.
\end{abstract}

\section{Keywords}

External Noise Array, Liquid Gallium, Microchannel, Taguchi Array.

\section{INTRODUCTION}

In recent times, the demand for efficient cooling solutions for emerging microelectronic and miniaturized systems has gained significant attention. Among various proposed solutions, liquid cooling using microchannel has gained significant attention owing to their numerous advantages such as direct integration on substrate. Significant research has been carried in last decade which include both experimental and numerical studies. With water being used as cooling medium numerous optimization studies can be found in literature mainly based on thermal resistance networks [13]Recently, liquid metals have been proposed to further increase cooling performance owing to their high thermal conductivity [4]. Among various such coolants reviewed, liquid gallium and its alloys have been found to be most suitable [5].The works of Miner and Ghoshal show that very high heat transfer coefficients (of the order $10 \mathrm{~W} / \mathrm{cm}^{2} / \mathrm{K}$ )are possible using these coolants [6]. Series of experiments conducted by Liu et al. also showed superior performance of liquid metal coolants[7]. When designing a microchannel heat sink it is necessary to know the effect of each geometrical parameter (channel width, aspect ratio, substrate material, channel spacing etc.) on its performance which helps in making a robust as well optimized design. Despite several studies available in literature using water regarding optimization, different thermo-physical properties of liquid gallium necessitate such a study for such coolants. This ismainly attributed to different heat transfer mechanism in liquid metals which mainly due to high conductivity than due to developing thermally boundary layer in water. However, unlike previous optimization studies carried out using water, heat transferrelations for forced convection in ducts using liquid metals are not available to the best of author's knowledge. Hence design of experiments technique is used for such an analysis.

In this study, Taguchi orthogonal array has been used to study the effect of various parameters. For this external noise is introduced in certain parameters as explained by Phadke[8]to get the effect of replication. This is necessary because in usual computer experiments the output is same for each run for similar input conditions. Hence repetition doesn't hold any advantage. It is worth mentioning that this is one of the techniques to analyze computer experiments as other methods such as Kriging models, MARS, RBF etc. have also been used in literature and discussed in literature[9-11].

\section{ANALYSIS PROCEDURE}

This section deals with analysis procedure adopted in this study. This includes selection of appropriate array, geometrical parameters, experimental units (computational domain) and material properties.

\subsection{Computational Domain}

Figure 1 shows computational domain of single layer microchannel. Various parameters considered in this study are shown.

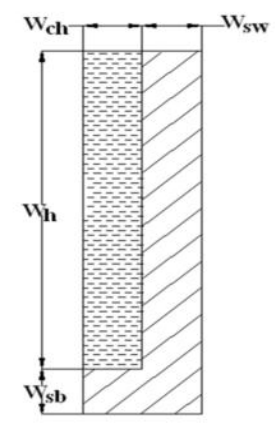

Fig 1: Computational Domain

$\mathrm{W}_{\mathrm{sb}}$ and $\mathrm{W}_{\mathrm{sw}}$ represents thickness of solid substrate from heated surface (base) and channel wall respectively. $\mathrm{W}_{\mathrm{sw}}$ mainly defines width of solid region which will help in deciding the number of channels for a given dimensions of heat sink. It is also referred as channel wall thickness in this study. $\mathrm{W}_{\mathrm{ch}}$ denotes half channel width while $\mathrm{W}_{\mathrm{h}}$ represents channel depth and their ratio being called as aspect ratio. Further two different substrate materials are considered whose properties along with those of fluid are given in Table 1. As 
per literature survey mentioned earlier, since liquid gallium is most suitable, it has been used in this study.

Table 1: Material Properties

\begin{tabular}{c|c|c|c}
\hline $\begin{array}{c}\text { Material } \\
\text { Properties }\end{array}$ & $\begin{array}{c}\text { Liquid } \\
\text { Gallium }\end{array}$ & $\begin{array}{c}\text { Aluminum } \\
\text { [Material1] }\end{array}$ & $\begin{array}{c}\text { Silicon } \\
\text { [Material 2] }\end{array}$ \\
\hline Density $\left(\mathrm{kg} / \mathrm{m}^{3}\right)$ & 6088 & 2719 & 2328 \\
\hline $\begin{array}{c}\text { Specific Heat } \\
(\mathrm{J} / \mathrm{kg}-\mathrm{K})\end{array}$ & 400 & 871 & 705 \\
\hline $\begin{array}{c}\text { Thermal } \\
\text { Conductivity } \\
(\mathrm{W} / \mathrm{m}-\mathrm{K})\end{array}$ & 29 & 202.4 & 150 \\
\hline $\begin{array}{c}\text { Viscosity } \\
(\mathrm{kg} / \mathrm{m}-\mathrm{s})\end{array}$ & 0.00187 & --- & --- \\
\hline
\end{tabular}

\subsection{Experiment Design}

Since the study involves 5 parameters, a $2^{7}$ Taguchi array is used for the analysis and various parameters are assigned to various columns as per requirement to study main or interaction effect. To study the interaction effect of $\mathrm{W}_{\mathrm{sb}}$ with substrate material and $\mathrm{W}_{\mathrm{sw}}$ with $\mathrm{W}_{\mathrm{sb}}$ columns 3 and 6 were left empty i.e. no parameter was assigned to these columns. The latter interaction effect was desired as heat flow from base to fluid is also governed by wall thickness. Various levels of each parameter are depicted in Table 2. Though in computation only half channel width is used, levels presented here are for full channel width.

Table 2: Various Parameters with Levels

\begin{tabular}{c|cc}
\hline Parameter & $\begin{array}{c}\text { Low } \\
\text { Level [1] }\end{array}$ & $\begin{array}{c}\text { High Level } \\
\text { [2] }\end{array}$ \\
\hline $\mathrm{W}_{\mathrm{sb}}$ & 50 & 100 \\
\hline $\mathrm{B}\left(\mathrm{W}_{\mathrm{sw}} / \mathrm{W}_{\mathrm{sb}}\right)$ & 0.5 & 1.5 \\
\hline $2 \mathrm{~W}_{\mathrm{ch}}$ & 100 & 200 \\
\hline $\begin{array}{c}\text { Aspect Ratio } \\
\left(\mathrm{AR}=\mathrm{W}_{\mathrm{h}} / 2 \mathrm{~W}_{\mathrm{ch}}\right)\end{array}$ & 1 & 3 \\
\hline $\mathrm{M}($ Material $)$ & 1 & 2 \\
\hline
\end{tabular}

The Taguchi Array along with parameters assigned to various columns is shown in Table 3. In this, levels corresponding to mean values (without noise) are shown.In microchannels, channel depth and width are of major concern as flow and thermal characteristics are mainly governed by their exact dimensions. Hence any deviation may lead to erroneous results or conclusions. Hence, external noise is introduced in these two variables i.e. channel width and depth.A 5\% deviation from mean is assumed at low level and $2.5 \%$ at higher level. This is due to the fact that with reduction in dimensions, it's more difficult to maintain same dimensions and there is greater probability of deviation from exact value.In above table DP No. is the design point number whereas $\mathrm{I} 1$ and $\mathrm{I} 2$ represent interaction effect of $\mathrm{W}_{\mathrm{sb}} * \mathrm{~B}$ and $\mathrm{W}_{\mathrm{sb}} * \mathrm{M}$ respectively.
Table 3: $2^{7}$ Taguchi Array with Parameters

\begin{tabular}{c|cccccccc}
\hline Rarameter & $\mathrm{W}_{\mathrm{sb}}$ & $\mathrm{B}$ & $\mathrm{I} 1$ & $2 \mathrm{~W}_{\mathrm{ch}}$ & $\mathrm{AR}$ & $\mathrm{I} 2$ & $\mathrm{M}$ \\
\hline 1 & & & & & & & & \\
\hline & 50 & 1 & 1 & 100 & 1 & 1 & 1 \\
\hline 2 & 50 & 1 & 1 & 200 & 3 & 2 & 2 \\
\hline 3 & 50 & 2 & 2 & 100 & 1 & 2 & 2 \\
\hline 4 & 50 & 2 & 2 & 200 & 3 & 1 & 1 \\
\hline 5 & 100 & 1 & 2 & 100 & 3 & 1 & 2 \\
\hline 6 & 100 & 1 & 2 & 200 & 1 & 2 & 1 \\
\hline 7 & 100 & 2 & 1 & 100 & 3 & 2 & 1 \\
\hline 8 & 100 & 2 & 1 & 200 & 1 & 1 & 2 \\
\hline
\end{tabular}

\subsection{Solution Method}

To analyze the performance of microchannels using liquid gallium (metal) as coolant, the continuity, momentum and energy equation are solved for fluid region along with energy equation in solid region using Finite Volume based commercial code FLUENT. Further since it is known that increasing flow rate affects the performance, hence the flow rate has been kept same in all the cases by adjusting the inflow velocity according to cross-sectional area. Grid independence study was performed and finally grid which on further refinement showed variation less than $1 \%$ was selected

\section{Results and Discussions}

The performance of a microchannel is mainly decided on the basis of thermal resistance, pump power and minimal temperature variations at the base. To comply with these parameters the following variables are used to evaluate the performance.

$\Delta \theta=\left(T_{\text {max }, \text { base }}-T_{\text {min,base }}\right)$

which represents temperature variations at the base and for superior performance it should be as low as possible.Here variables $T_{\text {max,base }}$ and $T_{\text {min,base }}$ represent maximum and minimum temperature attained at the heated surface (base). Similarly, the overall performance of microchannel which is governed by minimizing thermal resistance at the cost of minimum pump power can be expressed as:

$\gamma=\frac{1}{10^{8}} \times \frac{(q)}{P P\left(T_{\text {max }, \text { base }}-T_{\text {inlet }}\right)}$

This factor is defined per unit heated area of microchannel. In above relation $q, P P$ and $T_{\text {inlet }}$ denote heat flux, pump power and fluid inlet temperature respectively. A factor of $10^{8}$ has been used to scale large values obtained within suitable range. Since it is just a linear transformation, the results are not affected. A $2^{7}$ array comprising of 8 runs on introduction of external noise in two variables was run for 4 times for a single design point. For example for design point 1, combination of $\mathrm{W}_{\text {ch }}$ and $\mathrm{W}_{\mathrm{h}}$ used for analysis were $(47.5,105),(52.5,105)$, $(52.5,95)$ and $(52.5,105)$ owing to deviation of $5 \%$ at low level and $2.5 \%$ at high level. The responses $(\Delta \theta, \gamma)$ 
corresponding to each design point were measured. Table 4 show values of $\Delta \theta$ while those for $\gamma$ are depicted in Table 5. Here trial refers to simulation for a particular combination of width and depth at a given design point.

Table 4: Output $\Delta \theta$ for 4 Trials for 8 design Points

\begin{tabular}{c|cccc}
\hline Trial & $\mathbf{1}$ & $\mathbf{2}$ & $\mathbf{3}$ & $\mathbf{4}$ \\
\hline 1 & & & & \\
\hline 2 & 19.297 & 18.704 & 20.623 & 21.285 \\
\hline 3 & 33.136 & 32.938 & 34.328 & 34.557 \\
\hline 4 & 32.244 & 30.456 & 33.6 & 35.579 \\
\hline 5 & 40.649 & 40.08 & 41.981 & 42.587 \\
\hline 6 & 25.368 & 24.233 & 25.423 & 26.62 \\
\hline 7 & 35.684 & 35.153 & 38.719 & 39.304 \\
\hline 8 & 45.314 & 42.643 & 44.733 & 47.525 \\
\hline & & & & 63.609 \\
\hline
\end{tabular}

ANOVA was also carried out and result corresponding $\Delta \theta$ and $\gamma$ are shown in Table 6 and Table 7 respectively.

Table 5: Output $\gamma$ for 4 Trials for 8 design Points

\begin{tabular}{c|rrrr}
\hline Trial & \multicolumn{1}{|c}{ 1 } & $\mathbf{2}$ & $\mathbf{3}$ & $\mathbf{4}$ \\
DP No. & & & & \\
\hline 1 & & & & \\
& 65.71 & 66.41 & 61.49 & 61.09 \\
\hline 2 & 3513.68 & 3617.50 & 3606.88 & 3503.08 \\
\hline 3 & 39.33 & 40.82 & 37.74 & 36.51 \\
\hline 4 & 2846.54 & 2956.46 & 2930.57 & 2822.96 \\
\hline 5 & 281.72 & 308.89 & 306.59 & 280.03 \\
\hline 6 & 532.52 & 535.19 & 497.47 & 496.08 \\
\hline 7 & 151.82 & 170.11 & 168.33 & 150.51 \\
\hline 8 & 324.24 & 329.99 & 306.28 & 301.72 \\
\hline
\end{tabular}

\subsection{Main and Interaction effect of $W_{s b}$ and}

B

The effect of substrate base thickness as well as wall thickness on various parameters is discussed in this section. This thickness is mainly responsible for conduction resistance which is encountered before heat passes to fluid medium. Figure 2 shows main effect plot of $\mathrm{W}_{\mathrm{sb}}$ and $\mathrm{B}$ on $\Delta \theta$. It is observed that both these parameters have significant effect on $\Delta \theta$ which is of similar nature. Increase in value of $\Delta \theta$ with increase in value of both the parameters can be justified based on the fact that with increase in thickness of the substrate, conduction resistance increases thereby increasing maximum temperature and hence value of $\Delta \theta$. Further, it can be inferred that effect of $\mathrm{B}\left(\mathrm{W}_{\mathrm{sw}}\right)$ is more predominant as compared $\mathrm{W}_{\mathrm{sb}}$ which is supported by ANOVA of $\Delta \theta$ in Table 6 .

Table 6: ANOVA for $\Delta \theta$

\begin{tabular}{c|rrrrc}
\hline Variable & DF & SS Total & MS & F value & P \\
\hline $\mathrm{W}_{\mathrm{sb}}$ & 1 & 783.41 & 783.41 & 213.54 & 0 \\
$\mathrm{~B}$ & 1 & 1979.4 & 1979.4 & 539.54 & 0 \\
$\mathrm{I} 1$ & 1 & 236.86 & 236.86 & 64.56 & 0 \\
$\mathrm{~W}_{\mathrm{ch}}$ & 1 & 1189.82 & 1189.82 & 324.32 & 0 \\
$\mathrm{AR}$ & 1 & 10.34 & 10.34 & 2.82 & 0.106 \\
$\mathrm{I} 2$ & 1 & 2.76 & 2.76 & 0.75 & 0.394 \\
$\mathrm{M}$ & 1 & 35.82 & 35.82 & 9.76 & 0.005 \\
\hline Error & 24 & 88.05 & 3.67 & & \\
\hline Total & 31 & 4326.46 & & & \\
\hline
\end{tabular}

Table 7: ANOVA for $\gamma$

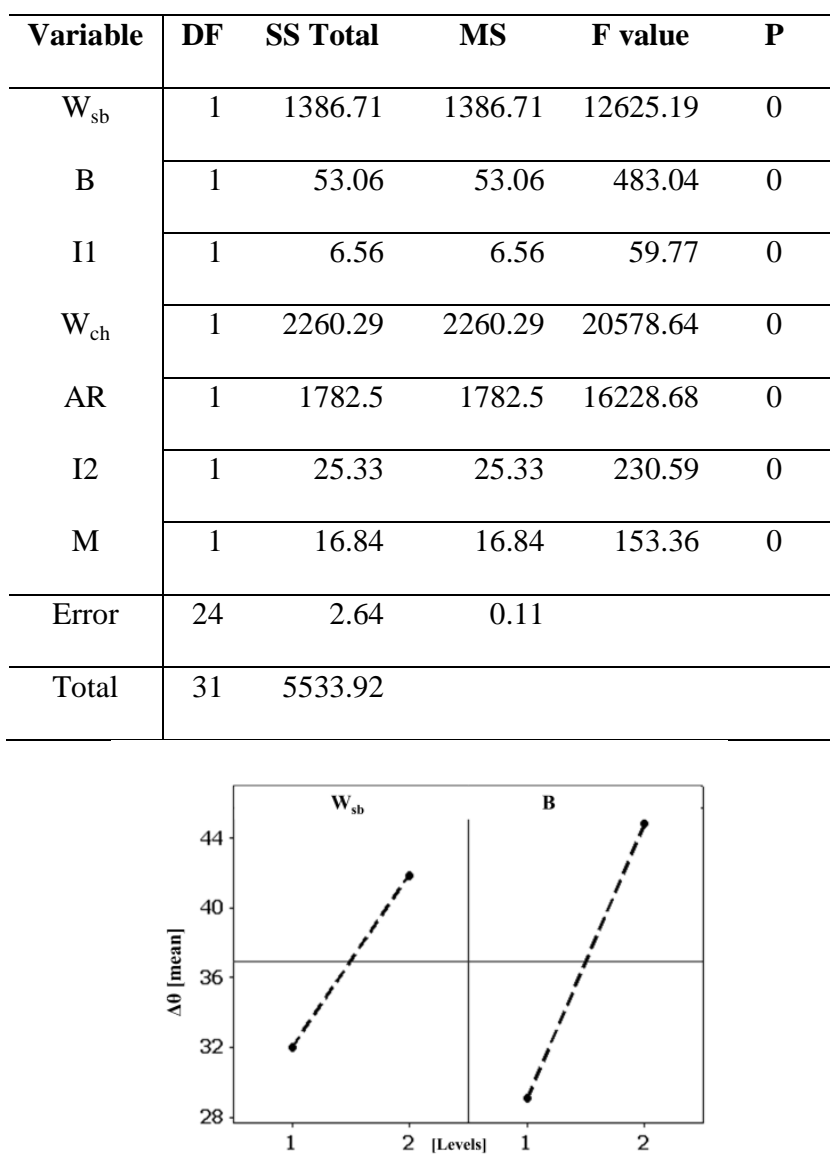

Fig 2: Main effect of $W_{s b}$ and $B$

Interaction effect of both these parameters is shown in Figure 3 along with main effect of I1. It is observed that there exists interactive behavior of both these parameters which influence 
$\Delta \theta$ and is justified by individual interaction plot. However, on comparing it with Figure 2, it can be deduced that the effect is less significant in comparison to main effects but cannot be neglected. These conclusions can be further verified from ANOVA Table 6 which shows significant effect of these two parameters as well as their interaction on temperature variation.
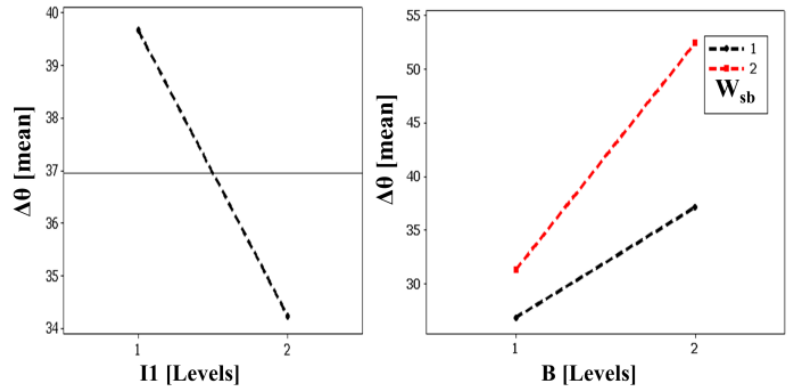

Fig 3: Main effect $I 1$ and interaction effect $W_{c h} * B$

Table 7 shows the effect of these parameters on the overall performance of microchannel. It is observed that base substrate thickness has significant effect on overall performance. This can be attributed to its contribution to thermal resistance. Also increase in $\mathrm{W}_{\mathrm{sb}}$ for a given total height of channel results in increase pump power which deteriorates the performance. This aspect can be clearly observed from main effect plot of $\mathrm{W}_{\mathrm{sb}}$ on $\gamma$ as shown in Figure 4.

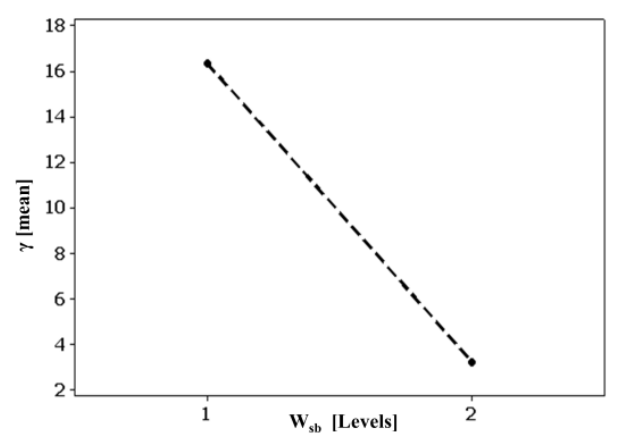

Fig 4: Main effect of $W_{s b}$ on overall performance parameter

Since lower value of substrate thickness from base offers lower conduction thermal resistance as well as results in lower pressure drop for fixed total height, superior overall performance as observed from Figure 4 can be explained at its lower level.

\subsection{Effect of Channel Width $\left(W_{\text {ch }}\right)$ and Depth $\left(\mathbf{W}_{\mathrm{h}}\right)$}

It is well known that channel width and depth constitute flow region and hence are the prime factors affecting the pumping power and thermal resistance in case of heat transfer through ducts. Channel depth can be calculated using aspect ratio which is considered as one of the parameter in this study. In theoretical terms, one can increase channel depth to any desired value for best performance. However, practical limitations such as space and pump power constraints limits its value. Table 6 shows that effect of channel width is quite significant in comparison to its depth $\Delta \theta$. This can be explained by the fact that width of channel governs immediate area from which heat transfer to cooling medium will take place. In case of heat flow through channel wall (which is governed by depth of channel) heat has to traverse longer path and hence more resistance. The effect of both these parameters on overall performance of microchannel with liquid gallium as coolant is found to be most significant as can be deduced from Table 7. This is further supported from Figure 5 which shows main effect of channel widthand depth on $\gamma$. It can be inferred that with increase in value of both these parameters, overall performance microchannel increases.

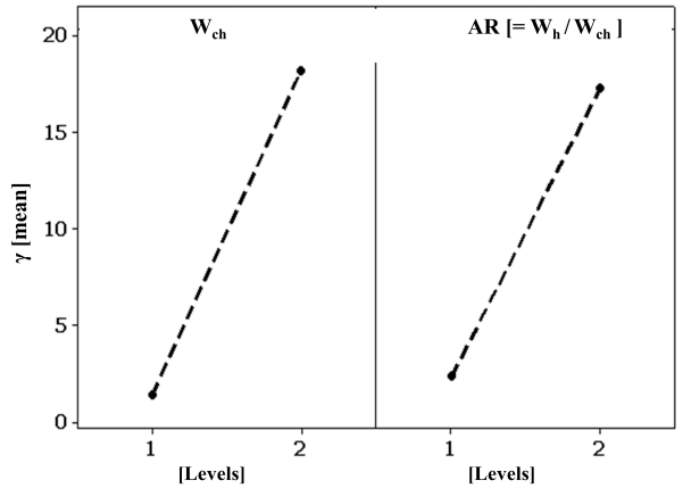

\section{Fig 5: Main effect of channel depth and aspect ratio on overall performance}

This can be explained as follows. Increase in channel width and depth corresponds to increase in flow area and hence larger flow rate which will help in reducing thermal resistance. Even in case of same flow rate, as considered in this study, increase in both these parameters result in larger hydraulic diameter and hence lower pressure drop thereby decreasing the required pump power. Consequently, the overall performance of the microchannel increases which can be clearly seen in Figure 5.

\subsection{Effect of Substrate Material}

For flow in ducts, heat transfer is mainly dependent on fluid characteristics. However, material of the substrate is also of prime importance as it provides the first passage of heat flow and hence initial thermal resistance. A poorly conducting material may be of no use despite increasing flow rate to maximum permissible value. Usually metals or their conducting alloys are used as substrate materials but all have different properties and hence it becomes necessary to evaluate type of material.

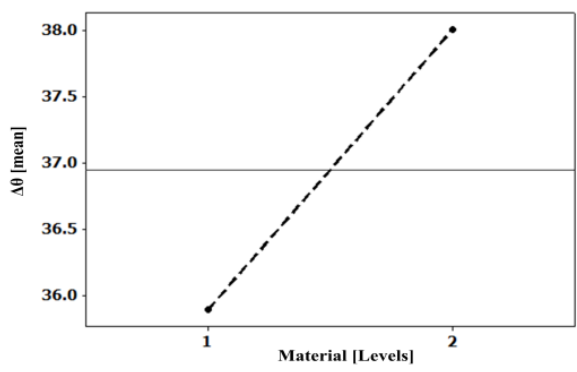

Fig 6: Main effect of parameter $M$ on $\Delta \theta$ 
As can be seen from ANOVA of both the parameters the type of substrate does hold importance at $95 \%$ significance level. However, in comparison of other parameters, their contribution is very less. Figure 6, shows main effect plot of substrate material on $\Delta \theta$. Since lower value of theta is desired, it is observed that material 1 is more suitable. Since we are mainly concerned as with its properties, it can be deduced that material with high thermal conductivity and heat capacity will help in reducing temperature variations as well as thermal resistance. This is further supported by the fact that higher thermal conductivity refers to lower thermal resistance and hence easy flow of heat from base to fluid thereby enhancing performance of microchannel. Another important observation is that interactive behavior of base thickness and type of material (I2) is negligible. This mainly represented thickness of a particular type of material. However, results show that this factor has negligible effect on both the parameters.

\section{OPTIMIZATION STUDY}

Till now, the effect of various parameters, has been analyzed and it has been found that channel depth, width and thickness of substrate from heated surface are the major factors affecting both the responses, Hence for a robust design it is necessary to know the values or trend towards which the parameters should move (increasing or decreasing) so as to get an optimum and robust design. In this section settings (values) for most important parameters obtained from ANOVA for both the parameters is discussed. It should be noted that settings described here are for the range of values considered in this study for a given parameter. In general, higher or lower value should be used as concluded in subsequent section. S/N (signal-to-noise) ratio for both these parameters is considered and plotted along with their mean effect as shown in Figure 7 and 8.

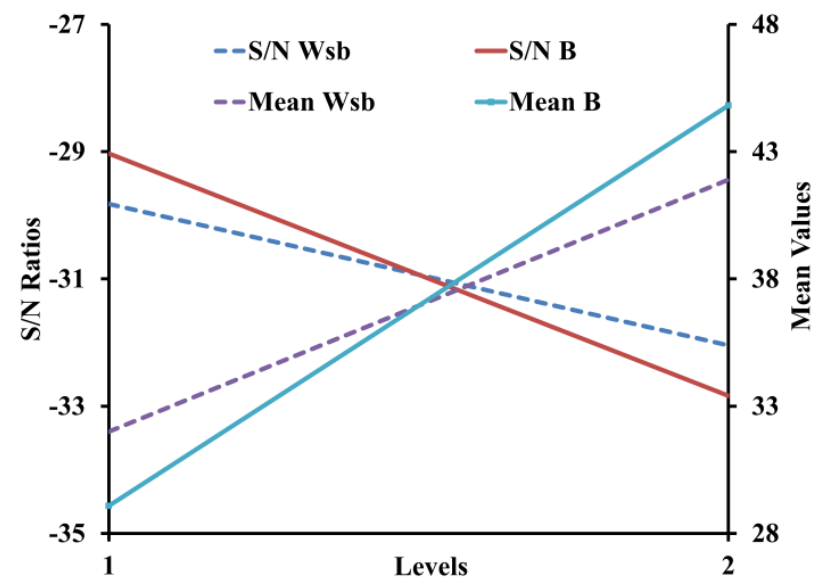

Fig 7: $\mathrm{S} / \mathrm{N}$ and Mean effect plot for $\Delta \theta$

It is to be noted that since minimum value if $\Delta \theta$ is desired, therefore minimum is the better relation is used whereas in case of $\gamma$ maximum is better relation is employed. For sake of brevity, results are plotted only for parameters having most significant effect as discussed above. As can be seen from Figure 7, for lower value of $\Delta \theta$ wall thickness as well as base substrate thickness should be set to low value. This is further supported from high $\mathrm{S} / \mathrm{N}$ values obtained at these settings. On the contrary, for $\gamma$ whose high value shows better performance, it can deduced from Figure 8 that channel width, depth and substrate base thickness should kept at higher value to obtain better performance. As can be clearly seen from higher $\mathrm{S} / \mathrm{N}$ values obtained at higher settings.

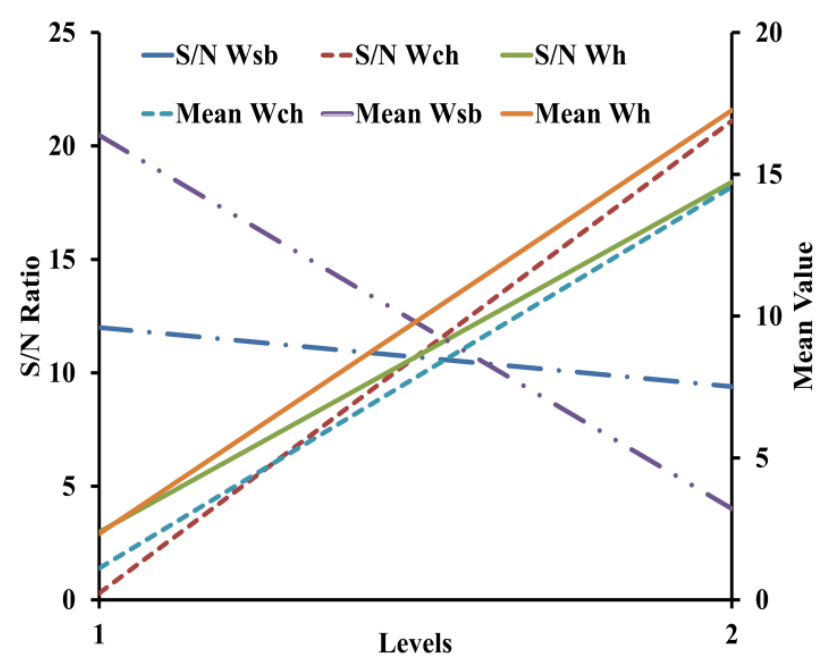

Fig 8: S/N and Mean effect plot for $\gamma$

\section{CONCLUSION}

Use of Taguchi Array with external noise is used to analyze the effect of various geometrical parameters on the performance of microchannel when using liquid gallium as cooling medium. It is observed that for minimum temperature variations at the heated surface, substrate thickness from the base, channel wall thickness as well as channel width is the most significant factors. In addition interaction of both these thickness also affects temperature variations at the base significantly. The overall performance of microchannel which incorporates both thermal resistance and pump power was defined by a parameter $\gamma$. It is observed that channel depth, width and substrate base thickness are the prime factors. For robust and optimum design higher values of width and depth along with lower value of base thickness is recommended. Apart from these the type of substrate material also affects the performance to some extent and the one with high values of thermal conductivity as well as specific heat should be used.

\section{ACKNOWLEDGMENTS}

The Authors will like to thanks Director CSIR-CSIO, Dr. PawanKapur for giving them opportunity to carry out this work. The authors also express their gratitude towards Dr. K. D. Chattopadhay for his guidance and support.

\section{REFERENCES}

[1] S. H. Chong, K. T. Ooi, and T. N. Wong.2002. "Optimisation of single and double layer counter flow microchannel heat sinks," Applied Thermal Engineering, vol. 22, pp. 1569-1585.

[2] X. Wei and Y. Joshi.2002. "Optimization study of stacked micro-channel heat sinks for micro-electronic cooling," in Thermal and Thermomechanical Phenomena in Electronic Systems, 2002. ITHERM 2002. The Eighth Intersociety Conference on, pp. 441-448.

[3] W. Xiaojin and Y. Joshi.2003. "Optimization study of stacked micro-channel heat sinks for micro-electronic cooling," Components and Packaging Technologies, IEEE Transactions on, vol. 26, pp. 55-61.

[4] J Liu and Y. X. Zhou.2002. China Patent No. 02131419.5 . 
[5] M. A. Kunquan and J. Liu.2007. "Liquid metal cooling in thermal management of computer chips," Frontiers of Energy and Power Engineering in China, vol. 1, pp. 384402.

[6] A. Miner and U. Ghoshal.2004. "Cooling of high-powerdensity microdevices using liquid metal coolants," Applied Physics Letters, vol. 85 pp. 506-508.

[7] T. Li, Y.-G. Lv, J. Liu, and Y.-X. Zhou.2005 "A powerful way of cooling computer chip using liquid metal with low melting point as the cooling fluid," Forschung im Ingenieurwesen, vol. 70, pp. 243-251.

[8] M. S. Phadke Quality Engineering Using Robust Design, Third ed.: Dorling Kindersley (Licensees of Pearson Education in South Asia).
[9] Timothy W. Simpson, Timothy M. Mauery, John J. Korte, and F. Mistree.1998. "Comparison of Response Surface Method and Kriging Modelsfor Multidisciplinary Design Optimization," in AIAA paper 98-4758 7 th AIAA/USAF/NASA/ISSMO Symposium on Multidisciplinary Analysis and Optimization (1998), pp. 98-4755.

[10] R.Jin, W.Chen, and T.W.Simpson.2000. "Comparative studies of metamodelling techniques under multiple modelling criteria," Structural and Multidisciplinary Optimization, vol. 23, pp. 1-13.

[11] Dudy Lim, Yew-Soon Ong, and Y. Jin.2007. "A Study on Metamodelling Techinuqes,Ensembles, and MultiSurrogates in Surrogate-Asssisted Memetic Algorithms," in 9th Annual Conference on Genetic and Evolutionary Computation (GECCO), London, pp. 1288-1295. 